\title{
Sociodemographic Characteristics of Women Able to Obtain Medication Abortion Before and After Ohio's Law Requiring Use of the Food and Drug Administration Protocol
}

\author{
Ushma D. Upadhyay,, Nicole E. Johns, Alice F. Cartwright, and Tanya E. Franklin²
}

\begin{abstract}
Purpose: In 2011, a law went into effect in Ohio that regulates how abortion care providers can offer medication abortion to their patients. We sought to evaluate changes in sociodemographic characteristics of Ohio medication abortion patients before and after the implementation of this law.

Methods: We used a retrospective cohort design, comparing characteristics of women obtaining a medication abortion at four abortion facilities before and after the law. We used chart data from January 2010 to January 2011 and February 2011 to October 2014. For any significant changes in sociodemographics found before and after the law, we used stratified cross-tabulations to disentangle whether they were likely related to the restricted gestational limit imposed by the law (lowered from 9 to 7 weeks gestation), or whether they were likely related to other burdens brought on by the law, such as increased costs and visits.

Results: Women obtaining a medication abortion after the law were more likely to be older $(p=0.01)$, have higher levels of education ( $p<0.001)$, be of white race $(p<0.001)$, have private insurance $(p=0.001)$, have no children $(p=0.002)$, and reside in a higher income zip code $(p=0.03)$. Both the reduced gestational limit and the increased costs and visits likely contributed to declines among black women and women with lower levels of education. The reduced gestational limit for medication abortion likely contributed to a decline among younger women and Medicaid recipient groups. The increased costs and visits imposed by the law likely contributed to the decline in medication abortion among women with no insurance and women with children.

Conclusion: The lower gestational limit, higher cost, and time and travel burdens exacted by Ohio's medication abortion law were associated with disproportionate reductions in medication abortion among the most disadvantaged groups. The law was associated with reduced access among women who were younger, of black race, less educated, and in lower socioeconomic groups.
\end{abstract}

Keywords: abortion; health policy; medication abortion; Ohio; sociodemographic characteristics

\section{Introduction}

Medication abortion is a nonsurgical abortion in which two medications, mifepristone (brand name: Mifeprex) and misoprostol, are taken $24-48 \mathrm{~h}$ apart to induce an abortion. The mifepristone is an antiprogestin that stops the pregnancy from continuing, and the misoprostol causes the cervix to soften and the uterus to contract, resulting in the expulsion of the contents of the uterus. The physical process of having a medication abortion is similar to a miscarriage. While abortion

${ }^{1}$ Advancing New Standards in Reproductive Health (ANSIRH), Bixby Center for Global Reproductive Health, Department of Obstetrics, Gynecology, and Reproductive Sciences, University of California, San Francisco, Oakland, California.

${ }^{2}$ Department of Obstetrics, Gynecology, and Women's Health, University of Louisville School of Medicine, Louisville, Kentucky.

*Address correspondence to: Ushma D. Upadhyay, PhD, MPH, Advancing New Standards in Reproductive Health (ANSIRH), Bixby Center for Global Reproductive Health, Department of Obstetrics, Gynecology, and Reproductive Sciences, University of California, San Francisco, 1330 Broadway, Suite 1100, Oakland, CA 94612, E-mail: ushma.upadhyay@ucsf.edu

(C) Ushma D. Upadhyay et al. 2018; Published by Mary Ann Liebert, Inc. This Open Access article is distributed under the terms of the Creative Commons License (http://creativecommons.org/licenses/by/4.0), which permits unrestricted use, distribution, and reproduction in any medium, provided the original work is properly cited. 
with pills is sometimes called a medical abortion, and was previously called RU-486, the term medication abortion most accurately represents the use of drugbased methods that can terminate pregnancy. ${ }^{1}$

The method was developed in France and approved there in $1988 .^{2}$ It underwent a 54-month politically charged review in the United States, and was ultimately approved by the Food and Drug Administration (FDA) in $2000 .^{2,3}$ The 2000 FDA protocol recommended its use only up to 7 weeks of pregnancy, involving a higher dose (600 $\mathrm{mg}$ ) of the more expensive drug, mifepristone, and a lower dose $(400 \mathrm{mcg})$ of the cheaper drug, misoprostol. For those who wanted a medication abortion, the 2000 FDA protocol required patients to visit a provider three times: first to take the mifepristone pills, second to take the misoprostol pills, and third for a follow-up visit to ensure the abortion was complete. This protocol is $92 \%$ effective only up to 7 weeks gestation. ${ }^{4}$

In the subsequent years after the initial registration of mifepristone in 2000, research and clinical practice demonstrated that an updated evidence-based protocol was more convenient, and more effective than the originally approved FDA protocol, with an effectiveness rate of 95-99\% when administered up to 9 weeks gestation. ${ }^{5-7}$ The evidence-based protocol permitted use up to 9 weeks of pregnancy, involving a lower dose $(200 \mathrm{mg})$ of the expensive mifepristone and a higher dose of cheaper misoprostol $(800 \mathrm{mcg})$. But probably the largest change from a patient perspective is that it eliminated a visit by not requiring patients to return to take the second set of pills, the misoprostol, in front of the provider. Research had demonstrated that women could be safely entrusted to take them at home ${ }^{8,9}$ according to instructions, although a follow-up visit was still usually required.
The 2010 National Abortion Federation guidelines recommended the evidence-based protocol. ${ }^{10}$ By 2011, most providers in the United States were no longer using the FDA protocol, and instead were using the evidence-based protocol. ${ }^{11}$

However, in 2011 a law went into effect in Ohio that regulated how abortion care providers could offer medication abortion to their patients. At the time, this law required clinicians to provide medication abortion according to the Food and Drug Administration protocol, as it was originally approved in 2000 which was still the official label (Table 1). Since in Ohio, all abortion patients must have a mandated information visit, followed by a 24 -h waiting period before they can obtain the mifepristone, providing care according to the 2000 protocol increased the total number of required visits to four.

In March 2016, the FDA updated the recommended protocol for Mifeprex to bring it in line with the clinical evidence base. These updates included expanding eligibility from 7 to 10 weeks gestation. It reduced the number of clinical office visits required from three visits to one; women could now obtain both medications in a single visit and take the first pills (mifepristone) to start their abortion when they wanted instead of at the doctor's office when they were able to get an appointment. The protocol no longer required patients to have an in-office follow-up appointment, allowing for alternatives to the follow-up visit. ${ }^{12}$ Thus, the new March 2016 protocol required only one visit for a medication abortion (but still two in Ohio with the mandated information visit).

Our previous research has demonstrated that after the 2011 Ohio law was enacted, women obtaining

Table 1. Protocol Comparison

\begin{tabular}{|c|c|c|}
\hline & Evidence-based regimen & $\begin{array}{l}\text { Original FDA-approved regimen } \\
\text { (as approved in 2000) }\end{array}$ \\
\hline Dates in use in study data & January 2010-January 2011 & February 2011-March 2016 \\
\hline Maximum days gestation & 9 weeks from LMPa & 7 weeks from LMP \\
\hline Mifepristone dose & $200 \mathrm{mg}$ orally in office & $600 \mathrm{mg}$ orally in office \\
\hline Misoprostol dose & 800 mcg vaginally or buccally (four tablets) & 400 mcg orally (two tablets) \\
\hline Misoprostol timing & $6-72 \mathrm{~h}$ after mifepristone & $48 \mathrm{~h}$ after mifepristone \\
\hline Misoprostol location & Home & Provider's office \\
\hline Follow-up visit & 5-14 days after mifepristone & 14 days after mifepristone \\
\hline Cost & Lower & Higher $^{b}$ \\
\hline $\begin{array}{l}\text { Minimum number of office visits (including Ohio's } \\
\text { required information visit) }\end{array}$ & 3 & 4 \\
\hline Efficacy rate & $95-99 \%$ up to 9 weeks gestation & $92 \%$ up to 7 weeks gestation \\
\hline
\end{tabular}


medication abortions were three times as likely to require additional treatments to complete their abortions compared with women before the law went into effect. ${ }^{13}$ We also found that the proportion of medication abortion patients at four facilities in the state declined dramatically, from $22 \%$ of all abortions in 2010 down to $5 \%$ in 2014 . There were no other changes in the provision of care or insurance acceptance during the same period at these facilities. State-level data confirm this finding: medication abortion fell significantly from $21 \%$ of all abortions in the state in 2010 to $5 \%$ of abortions in $2011(p<0.001)$ where it remained through 2014 (Fig. 1). ${ }^{14-18}$ In addition, other statewide analyses of mifepristone use between the years 2004-2014 in four states with large populations of women of reproductive age (including Ohio) corroborate massive declines in mifepristone use when the laws went into effect in Ohio and Texas, compared with steady and continued increases in mifepristone use in two states that did not have any restrictions on medication abortion (California and New York). ${ }^{19}$ Further national data from the same period on nonhospital abortions demonstrate a steady increase in the proportion of medication abortions from $17 \%$ in 2008 to $24 \%$ in 2011 to $31 \%$ in $2014 . .^{20,21}$

Having the option of medication abortion is important to many women seeking abortion. Some women prefer medication abortion for several reasons: so they can be at home for the abortion, avoid aspiration, surgery, and/or anesthesia, and because they believe the method to be less invasive, painful, or frightening, or more natural or safer. ${ }^{22,23}$ When women are offered a choice between medication abortion and aspiration, they find the method they personally choose for themselves to be highly acceptable. ${ }^{24,25}$ However, there is no evidence demonstrating that sociodemographic characteristics affect patient preferences between medication abortion and aspiration abortion. ${ }^{22,26}$ Research has consistently shown that medication abortion is highly acceptable to women from diverse racial/ethnic groups, education levels, and income levels. ${ }^{22,26-29}$

Due to factors intersecting at the individual, societal, and health system levels, ${ }^{30}$ women of color and low-income women are disproportionately represented among abortion patients relative to the general population in the United States. Black and Hispanic women are overrepresented, and three-quarters of abortion patients are low income $(<200 \%$ of the federal poverty level). ${ }^{31,32}$ One of the reasons for high relative rates of abortion is that rates of unintended pregnancies are higher among populations of women of color, low-income women, and women with lower educational attainment. ${ }^{33,34}$ Differences in rates of unintended pregnancy are related to disparities in rates of contraceptive use. Systematic barriers to contraceptive access such as lack of health insurance coverage or high out-of-pocket costs for certain methods can make contraception out of reach for many women. In addition, concerns about safety and fears of coercion related to hormonal contraception and sterilization (more effective methods) ${ }^{35}$ stem from the history of discriminatory practices directed at controlling and devaluing

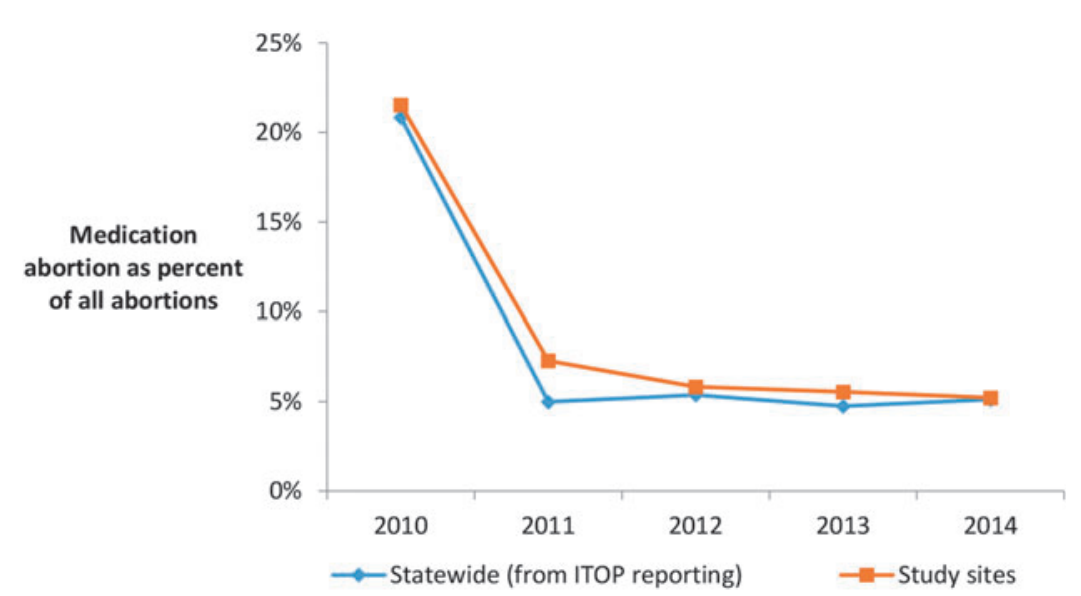

FIG. 1. Medication abortion as a percentage of all abortions in Ohio 2010-2014, study clinic and overall state data. State data from Ohio Department of Health ITOP statistics. ITOP, Induced Termination of Pregnancy. 
the fertility of low-income women and women of color. $^{36-39}$

In our previous research, we found that the sociodemographic characteristics of women who had medication abortions under 7 weeks gestation changed significantly following the implementation of the FDA protocol requirement. ${ }^{13}$ However, that analysis was unable to determine whether the changes in sociodemographic characteristics were only because it excluded women 7-9 weeks gestation, or whether the barriers imposed by the law systematically prevented certain groups of women from having a medication abortion. In this analysis, we look at all women who were able to obtain a medication abortion before and after the law, regardless of weeks gestation, to determine the impact of the law by sociodemographic characteristics.

\section{Materials and Methods}

We abstracted medical chart data at four abortionproviding facilities in Ohio for all medication abortions from 1 year prior to the law's implementation (January 2010-January 2011) to 3 years postimplementation (February 2011-October 2014). We chose to abstract 1 year of prelaw data and 3 years of postlaw data because after the law went into effect, the number of medication abortions dropped to such a degree that we needed to collect 3 years of data to reach the $a$ priori calculated sample size required to achieve sufficient statistical power. The Institutional Review Board of the University of California, San Francisco, granted ethical approval for this study. The data collection methodology has been previously described. ${ }^{13}$ Abstraction occurred between September 2014 and April 2015.

\section{Measures}

Sociodemographic measures abstracted from patient charts included age, highest level of education, race/ ethnicity, insurance status, zip code, height, weight, and previous births. (In Ohio, Medicaid cannot be used to pay for abortion, and thus insurance status did not necessarily reflect how the patient paid for the abortion; we use it as a proxy for socioeconomic status.) Distance traveled to abortion care was calculated based on home zip code (the most detailed location information available) to facility using the "traveltime3" STATA module, which utilizes a Google Maps application programming interface to calculate driving distance. Clinical information beyond the consent visit was not abstracted for women at gestations 7-9 weeks in the prelaw period, so we used gestation at the consent visit rather than gestation at the mifepristone visit. Previous births were used as an imperfect proxy for having children. When missing, the number of previous births was computed based on the number of previous vaginal births, number of previous cesarean sections, number of previous pregnancies, number of previous abortions, and number of previous miscarriages. Zipcode-based household income quintile information was obtained from 2010 census data via the Michigan Population Studies Center. ${ }^{40}$ All other data were analyzed as they appeared in the chart.

\section{Data analysis}

We performed the analysis in two parts: First, we compared characteristics of the overall study population obtaining a medication abortion at the four study sites before and after the law. We compared the distributions of sociodemographic characteristics of women able to obtain a medication abortion at any gestation by first examining results from overall chi-square tests. If the overall chi-square test was statistically significant, at $p<0.05$, we conducted group-wise $t$-tests to learn which group differences were statistically significant. In the text, we report the overall $p$-values for ordinal categorical variables and individual $p$-values for nominal categorical variables when statistically significant at $p<0.05$.

Second, we sought to further disentangle whether any changes in sociodemographics found were likely related to the restricted gestational limit imposed by the law (lowered from 9 to 7 weeks gestation), or whether they were likely related to other factors impacted by the law, such as increased costs and visits. To do so, we repeated chi-square tests of distributions stratified by gestation, comparing those patients at $<7$ weeks gestation prelaw with those patients 7-9 weeks gestation prelaw (to examine whether differences were potentially associated with changing gestational limits) and comparing those patients at $<7$ weeks gestation prelaw with those patients $<7$ weeks gestation postlaw (to examine differences potentially associated with law-related factors other than gestational limits). We report chi-square and $t$-test results similarly to the analysis above. All statistical analyses were conducted using Stata version 14.0.

\section{Results}

The decline in medication abortion was not evenly distributed across sociodemographic groups; we found significant differences in the characteristics of women obtaining medication abortion before and after the law (Table 2). As a direct result of the law which lowered 
Table 2. Characteristics of the Population Obtaining a Medication Abortion Among Patients from Four Abortion-Providing Facilities in Ohio, 2010-2014

\begin{tabular}{|c|c|c|c|c|}
\hline & Prelaw & Postlaw & Total & $\begin{array}{c}\text { Significance } \\
\text { prelaw vs. } \\
\text { postlaw }^{\text {a }}\end{array}$ \\
\hline$N$, numbers & 2169 & 1627 & 3796 & \\
\hline Age, $\%$ & & & & 0.01 \\
\hline$<20$ & 15.4 & 12.0 & 13.9 & 0.003 \\
\hline $20-24$ & 35.8 & 34.4 & 35.2 & N.S. \\
\hline $25-29$ & 23.9 & 26.0 & 24.8 & N.S. \\
\hline $30-39$ & 21.9 & 24.2 & 22.9 & N.S. \\
\hline $40+$ & 3.0 & 3.4 & 3.2 & N.S. \\
\hline Highest level of education, $\%$ & & & & $<0.001$ \\
\hline $\begin{array}{l}\text { Less than high school } \\
\text { degree }\end{array}$ & 11.9 & 7.6 & 10.0 & $<0.001$ \\
\hline $\begin{array}{l}\text { High school diploma } \\
\text { or GED }\end{array}$ & 40.8 & 35.5 & 38.5 & 0.001 \\
\hline $\begin{array}{l}\text { Associates degree/some } \\
\text { college }\end{array}$ & 25.1 & 29.4 & 27.0 & 0.004 \\
\hline $\begin{array}{l}\text { Bachelors degree or } \\
\text { higher }\end{array}$ & 13.6 & 23.2 & 17.7 & $<0.001$ \\
\hline Not in chart & 8.7 & 4.3 & 6.8 & $<0.001$ \\
\hline Race/ethnicity, \% & & & & $<0.001$ \\
\hline White & 65.2 & 71.3 & 67.8 & $<0.001$ \\
\hline Black & 23.5 & 15.7 & 20.2 & $<0.001$ \\
\hline Latina & 3.8 & 4.6 & 4.1 & N.S. \\
\hline Asian/Pacific Islander & 3.1 & 3.9 & 3.5 & N.S. \\
\hline Other/not in chart & 4.4 & 4.5 & 4.4 & N.S. \\
\hline Insurance, \% & & & & $<0.001$ \\
\hline Private & 23.2 & 33.7 & 27.7 & $<0.001$ \\
\hline Medicaid/Medicare & 19.9 & 17.1 & 18.7 & 0.03 \\
\hline None & 28.7 & 24.4 & 26.8 & 0.003 \\
\hline Not in chart & 28.2 & 24.8 & 26.7 & 0.02 \\
\hline Distance traveled for care, \% & & & & N.S. \\
\hline$<50$ miles & 86.5 & 85.7 & 86.2 & \\
\hline $50+$ miles & 12.1 & 13.4 & 12.6 & \\
\hline Not in chart & 1.4 & 0.9 & 1.2 & \\
\hline Urban/rural, \% & & & & N.S. \\
\hline Urban & 93.5 & 93.4 & 93.5 & \\
\hline Rural & 5.3 & 5.9 & 5.6 & \\
\hline Not in chart & 1.2 & 0.7 & 1.0 & \\
\hline BMI category, \% & & & & N.S. \\
\hline Underweight $(<18.5)$ & 3.5 & 3.6 & 3.5 & \\
\hline Healthy weight (18.5-25) & 45.8 & 51.6 & 48.3 & \\
\hline Overweight $(25-30)$ & 24.7 & 23.5 & 24.2 & \\
\hline Obese (30-35) & 9.1 & 9.1 & 9.1 & \\
\hline Morbidly obese (35+) & 7.5 & 7.0 & 7.3 & \\
\hline Not in chart & 9.4 & 5.2 & 7.6 & \\
\hline Gestation at consent, $\%$ & & & & $<0.001$ \\
\hline Up to 4 weeks 6 days & 10.7 & 19.4 & 14.5 & $<0.001$ \\
\hline $\begin{array}{l}5 \text { weeks } 0 \text { day to } \\
5 \text { weeks } 6 \text { days }\end{array}$ & 22.1 & 45.4 & 32.1 & $<0.001$ \\
\hline $\begin{array}{c}6 \text { weeks } 0 \text { day to } \\
7 \text { weeks } 0 \text { day }\end{array}$ & 33.0 & 35.2 & 33.9 & N.S. \\
\hline $\begin{array}{c}7 \text { weeks } 1 \text { day to } \\
9 \text { weeks } 0 \text { day }\end{array}$ & 31.6 & 0.0 & 18.1 & $<0.001$ \\
\hline Not in chart & 2.6 & 0.0 & 1.5 & $<0.001$ \\
\hline Previous births, $\%$ & & & & 0.002 \\
\hline 0 & 49.5 & 54.7 & 51.7 & 0.002 \\
\hline 1 or more & 49.9 & 44.9 & 47.8 & 0.002 \\
\hline Not in chart & 0.6 & 0.4 & 0.5 & N.S. \\
\hline
\end{tabular}

(continued)
Table 2. Continued

\begin{tabular}{lrrrc}
\hline & & & $\begin{array}{c}\text { Significance } \\
\text { prelaw vs. } \\
\text { postlaw }\end{array}$ \\
\hline Zip-code-based national household income quintile, \% & 0.03 \\
$0-20$ & 1.9 & 1.2 & 1.6 & N.S. \\
$20-40$ & 18.3 & 17.4 & 17.9 & N.S. \\
$40-60$ & 54.4 & 52.2 & 53.4 & N.S. \\
$60-80$ & 23.1 & 26.8 & 24.7 & 0.01 \\
$80-100$ & 0.9 & 1.4 & 1.1 & N.S. \\
Not in chart & 1.5 & 1.0 & 1.3 & N.S. \\
\hline
\end{tabular}

${ }^{a} p$-Values for overall characteristic chi-square tests based on in-chart data only.

BMI, body mass index; GED, general educational development; N.S., pre- and postlaw differences not statistically significant.

the legal gestational limit for medication abortion from 9 to 7 weeks, women in the postlaw period had their medication abortions at significantly earlier gestations $(p<0.001)$. In the prelaw period, about one-third of the medication abortions were occurring between 7 and 9 weeks gestation. This dropped to $0 \%$ in the postlaw period as a result of the law.

The population obtaining a medication abortion in the postlaw period was significantly more likely to be older $(p=0.01)$, have higher levels of education $(p<0.001)$, be of white race $(p<0.001)$, have private insurance $(p<0.001)$, and have no children $(p=0.002)$ than women obtaining medication abortion before the law went into effect. In addition, in the post law period this population was significantly less likely to be of black race $(p<0.001)$, and less likely to have Medicaid $(p=0.03)$ or no health insurance at all $(p=0.003)$ compared with women in the prelaw period. The distribution by body mass index did not change significantly after the law. Residence-related measures did not show as much change; there was no significant change in distance traveled or urban/rural residence before and after the law, but women obtaining medication abortion after the law were less likely to reside in a lowincome zip code (one of the bottom two quintiles of household wealth, overall $p=0.03$ ).

When we compared populations at $<7$ weeks prelaw and 7-9 weeks prelaw as well as populations $<7$ weeks pre- and postlaw, a few characteristics were significantly different for both comparisons, namely race/ethnicity and education (Table 3). This suggests that changes in the distributions of women by race/ethnicity and education were potentially driven by changes in the gestational limit and by other factors related to the law. In the prelaw period, black women were more heavily represented in 
the population obtaining abortions at 7-9 weeks (27\%) than those obtaining abortions at $<7$ weeks $(21 \%$, $p<0.001$ ), so the gestational limit change would be expected to affect the number of black women obtaining medication abortions. In addition, limiting the comparison between pre- and postlaw distributions to those obtaining abortions at $<7$ weeks, there is still a significantly lower representation of black women in the postlaw period $(16 \%)$ than the prelaw period $(21 \%, p<0.001)$. Controlling for gestation by looking at women only $<7$ weeks, there appears to be an association between other factors related to the law and black women's ability to obtain an abortion.

Similarly, in the prelaw period, women with lower levels of education (less than high school and high school/general educational development) were more heavily represented in the population obtaining abortions at 7-9 weeks than those obtaining abortions at $<7$ weeks (overall $p<0.001$ ). Comparing pre- and postlaw distributions of women obtaining abortions at $<7$ weeks, we find significantly lower representation of women with less than high school in the postlaw period (overall $p<0.001$ ). These results suggest that this change was associated with both the change in gestational limit and other factors imposed by the law, such as increased visits and costs.

The age distribution was significantly different between women at $<7$ weeks and those at $7-9$ weeks gestation in the prelaw period but not for women at gestations $<7$ weeks before and after the law. Similarly, in the prelaw period women with Medicaid are overrepresented in the population obtaining abortions at 7-9 weeks (23\%) compared with those $<7$ weeks (17\%), but there was no difference among women $<7$ weeks pre- and postlaw. These comparisons suggest that the shift toward older age and reductions in Medicaid recipients after the law were potentially driven by the restriction to earlier gestations under the law.

In contrast, we see that there is no significant difference in the percentage of patients with no health insurance $<7$ weeks and 7-9 weeks before the law; however, there was a statistically significant drop in the proportion of women with no health insurance $<7$ weeks after the law $(30-24 \%, p<0.001)$. Similarly, there was no difference in the proportion of women with children obtaining an abortion $<7$ weeks and 7-9 weeks gestation in the prelaw period, but a difference among women at $<7$ weeks gestation prelaw $(50 \%)$ versus postlaw $(45 \%, p<0.006)$, suggesting that factors other than the gestational limit may have driven the shift toward fewer women with previous pregnancies (and existing children) obtaining medication abortion in the postlaw period.

\section{Discussion}

We found that a 2011 Ohio law requiring use of an outdated protocol for medication abortion appeared to have disproportionate effects on the most disadvantaged groups. The law seemed to affect younger women, black women, less educated women, and women with Medicaid or no health insurance most. The shift in the sociodemographic profile of medication abortion patients before and after the law is potentially driven by a combination of the lower gestational limit at which medication abortion was available and the increased logistical, financial, time, and travel burdens that the law exacted on women. Women were required to make four visits to the facilities instead of three, resulting in more transportation costs, time away from work and school, and increased need for childcare.

We were able to separate parts of the law that were associated with the declines in use among different sociodemographic subgroups. Both the reduced gestational limit and additional factors, such as logistical and financial burdens, appear to have contributed to the declines among black women and women with less education. The reduced gestational limit for medication abortion (and not the increased burdens) was associated with the decline among younger women, who are known to recognize their pregnancies later, ${ }^{41}$ and Medicaid recipient groups. Finally, the additional logistical and financial burdens imposed by the law (and not the decline in gestational limit) were associated with the decline in medication abortion among women with no insurance and women with children.

Laws requiring use of the FDA-approved protocol do not exist in isolation; they are present in states such as Ohio and Texas, which have many other abortion restrictions in place (e.g., Medicaid coverage restrictions, physician-only ultrasound laws, two-visit requirements, waiting periods). ${ }^{42}$ The cumulative burden of these many requirements may make the service logistically impossible for some women and providers. For others, the additional requirements may cause delays in care, resulting in women seeking abortions at later gestations. These women may have fewer options, becoming ineligible for medication abortion and pushed into later, more costly abortion procedures. While laws like the FDA-protocol restriction may not appear to cause undue burden on their own, their effects must 
Table 3. Characteristics of Populations Obtaining Medication Abortion at $<7$ Weeks Gestation Prelaw, 7-9 Weeks Gestation Prelaw, and <7 Weeks Gestation Postlaw at Four Abortion-Providing Facilities in Ohio, 2010-2014

\begin{tabular}{|c|c|c|c|c|c|c|}
\hline & \multicolumn{3}{|c|}{$\begin{array}{l}\text { Comparison of prelaw populations } \\
\text { by gestation (effect of law likely due } \\
\text { to lowered gestational limit) }\end{array}$} & \multicolumn{3}{|c|}{$\begin{array}{l}\text { Comparison of populations at }<7 \text { weeks } \\
\text { gestation by time period (effect of law } \\
\text { due to other burdens imposed by law) }\end{array}$} \\
\hline & $\begin{array}{c}\text { Prelaw } \\
<7 \text { weeks }\end{array}$ & $\begin{array}{c}\text { Prelaw } \\
\text { 7-9 weeks }\end{array}$ & $\begin{array}{l}\text { Significance: } \\
<7 \text { weeks vs. } 7-9 \\
\text { weeks prelaw }^{\mathrm{a}}\end{array}$ & $\begin{array}{c}\text { Prelaw } \\
<7 \text { weeks }\end{array}$ & $\begin{array}{c}\text { Postlaw } \\
<7 \text { weeks }\end{array}$ & $\begin{array}{l}\text { Significance: } \\
<7 \text { weeks prelaw vs. } \\
<7 \text { weeks postlaw }^{\mathrm{a}}\end{array}$ \\
\hline$N$, numbers & 1156 & 1013 & & 1156 & 1627 & \\
\hline Age, $\%$ & & & $<0.001$ & & & N.S. \\
\hline$<20$ & 14.3 & 16.7 & N.S. & 14.3 & 12.0 & \\
\hline $20-24$ & 33.3 & 38.7 & 0.009 & 33.3 & 34.4 & \\
\hline $25-29$ & 23.7 & 24.2 & N.S. & 23.7 & 26.0 & \\
\hline $30-39$ & 25.2 & 18.2 & $<0.001$ & 25.2 & 24.2 & \\
\hline $40+$ & 3.5 & 2.3 & N.S. & 3.5 & 3.4 & \\
\hline Highest level of education, \% & & & $<0.001$ & & & $<0.001$ \\
\hline Less than high school degree & 10.1 & 13.9 & 0.006 & 10.1 & 7.6 & 0.02 \\
\hline High school diploma or GED & 38.3 & 43.5 & 0.01 & 38.3 & 35.5 & N.S. \\
\hline Associates degree/some college & 27.7 & 22.2 & 0.003 & 27.7 & 29.4 & N.S. \\
\hline Bachelor's degree or higher & 14.9 & 12.0 & N.S. & 14.9 & 23.2 & $<0.001$ \\
\hline Not in chart & 9.0 & 8.3 & N.S. & 9.0 & 4.3 & $<0.001$ \\
\hline Race/ethnicity, \% & & & 0.001 & & & 0.001 \\
\hline White & 68.2 & 61.9 & 0.002 & 68.2 & 71.3 & N.S. \\
\hline Black & 20.7 & 26.8 & $<0.001$ & 20.7 & 15.7 & $<0.001$ \\
\hline Latina & 3.3 & 4.3 & N.S. & 3.3 & 4.6 & N.S. \\
\hline Asian/Pacific Islander & 3.8 & 2.3 & 0.04 & 3.8 & 3.9 & N.S. \\
\hline Other/not in chart & 4.1 & 4.7 & N.S. & 4.1 & 4.5 & N.S. \\
\hline Insurance, \% & $<0.001$ & & & $<0.001$ & & \\
\hline Private & 26.6 & 19.4 & $<0.001$ & 26.6 & 33.7 & $<0.001$ \\
\hline Medicaid/Medicare & 17.2 & 23.0 & $<0.001$ & 17.2 & 17.1 & N.S. \\
\hline None & 30.1 & 27.1 & N.S. & 30.1 & 24.4 & $<0.001$ \\
\hline Not in chart & 26.0 & 30.6 & 0.02 & 26.0 & 24.8 & N.S. \\
\hline Distance traveled for care, $\%$ & & & N.S. & & & N.S. \\
\hline$<50$ miles & 86.7 & 86.4 & & 86.7 & 85.7 & \\
\hline $50+$ miles & 12.1 & 12.0 & & 12.1 & 13.4 & \\
\hline Not in chart & 1.2 & 1.6 & & 1.2 & 0.9 & \\
\hline Urban/rural, \% & & & N.S. & & & N.S. \\
\hline Urban & 94.0 & 92.9 & & 94.0 & 93.4 & \\
\hline Rural & 4.9 & 5.7 & & 4.9 & 5.9 & \\
\hline Not in chart & 1.0 & 1.4 & & 1.0 & 0.7 & \\
\hline BMI category, \% & & & N.S. & & & N.S. \\
\hline Underweight $(<18.5)$ & 3.4 & 3.6 & & 3.4 & 3.6 & \\
\hline Healthy weight (18.5-25) & 47.5 & 43.9 & & 47.5 & 51.6 & \\
\hline Overweight $(25-30)$ & 25.9 & 23.4 & & 25.9 & 23.5 & \\
\hline Obese $(30-35)$ & 8.7 & 9.6 & & 8.7 & 9.1 & \\
\hline Morbidly obese (35+) & 8.0 & 7.0 & & 8.0 & 7.0 & \\
\hline Not in chart & 6.7 & 12.5 & & 6.7 & 5.2 & \\
\hline Previous births, \% & & & N.S. & 0.005 & & 0.005 \\
\hline 0 & 49.2 & 49.9 & & 49.2 & 54.7 & 0.004 \\
\hline 1 or more & 50.3 & 49.6 & & 50.3 & 44.9 & 0.006 \\
\hline Not in chart & 0.5 & 0.6 & & 0.5 & 0.4 & N.S. \\
\hline Zip-code-based national household income quintile, \% & & & N.S. & & & N.S. \\
\hline $0-20$ & 1.3 & 1.7 & & 1.3 & 1.2 & \\
\hline $20-40$ & 1.5 & 2.4 & & 1.5 & 17.4 & \\
\hline $40-60$ & 17.0 & 19.7 & & 17.0 & 52.2 & \\
\hline $60-80$ & 54.2 & 54.6 & & 54.2 & 26.8 & \\
\hline $80-100$ & 25.1 & 20.9 & & 25.1 & 1.4 & \\
\hline Not in chart & 1.0 & 0.7 & & 1.0 & 1.0 & \\
\hline
\end{tabular}

${ }^{a} p$-Values for overall characteristic chi-square tests based on in-chart data only.

be considered in conjunction with all restrictions on abortion present in a state.

We had only individual-level data on the population who obtained medication abortions. We did not have any data on the population who obtained aspiration or did not obtain an abortion at all. Therefore, we were unable to determine whether women who wanted but did not have a medication abortion in the postlaw period subsequently had an aspiration abortion or did not have an abortion at all. Indeed, research done at 
abortion clinics throughout Texas in 2014 which has a similar law found women whose nearest clinic had closed were more likely to have a "frustrated demand for medication abortion"-meaning that they reported a preference for medication abortion but ended up having or expecting to have an aspiration abortioncompared with women whose nearest clinic remained open. ${ }^{43}$

This study has a few limitations: This study was observational, relying on pre-/postlaw data with no control group of abortion patients who were not exposed to the law. Thus, causal relationships cannot definitely be drawn. However, the changes in the patterns of patients match exactly with the timing of the implementation of the law. In addition, data used in this study were abstracted from medical charts, allowing the potential for bias. We were unable to blind the abstractors to whether the chart was from the prelaw or postlaw period because the dates of visits were clearly in the charts. In an effort to reduce bias, each abstractor was given extensive training aimed at minimizing bias. In addition, we were lacking data on income, and used insurance coverage and zip code as proxies, limiting our ability to make conclusions on the effects of Ohio's law by income level.

Women have their own personal reasons for preferring medication abortion or aspiration abortion. Offering women a real choice between the two is a component of patient-centered care. State laws on specific types of abortion procedures limit women's ability to access the best and most acceptable healthcare for their situation. State-level restrictions do not affect all groups of women equally and can exacerbate inequities in access to abortion.

\section{Acknowledgments}

The authors appreciate study project management from Sarah L. Combellick, MPH, study support, and data cleaning from Elise Belusa, MSc and Anna Bernstein, MPH. They also wish to thank the staff at each facility who participated in the study for providing data and overall logistical administration, including Chrisse France, MEd, and Heather Harrington at Preterm, Sher'rie Brown, Kelli Halter, MS, Roslyn Kade, $\mathrm{MD}$, and Sharon Liner, MD, at Planned Parenthood Southwest Ohio, and Kate Huebner, MSW, Stephanie Kight, and Tim Kress, MD, and Michelle Meredith, CNP, at Planned Parenthood Greater Ohio. They also thank Adelaide Appiah, Bethany Lawrence, Brenna Lisowski, JD, Novneet Sandhu, Hannah Smith, MS, and Heather Tripp for chart abstraction. This study was supported by funding from a private foundation that wishes to remain anonymous.

\section{Author Disclosure Statement}

No competing financial interests exist.

\section{References}

1. Weitz TA, Foster A, Ellertson C, et al. "Medical" and "surgical" abortion: rethinking the modifiers. Contraception. 2004;69:77-78.

2. Winikoff $B$, Westhoff $C$. Fifteen years: looking back and looking forward. Contraception. 2015;92:177-178.

3. Greene MF, Drazen JM. A new label for mifepristone. N Engl J Med. 2016; 374:2281-2282.

4. Spitz IM, Bardin CW, Benton L, et al. Early pregnancy termination with mifepristone and misoprostol in the United States. N Engl J Med. 1998; 338:1241-1247.

5. Raymond EG, Shannon C, Weaver MA, et al. First-trimester medical abortion with mifepristone $200 \mathrm{mg}$ and misoprostol: a systematic review. Contraception. 2013;87:26-37.

6. American College of Obstetricians and Gynecologists. Practice bulletin no. 143: medical management of first-trimester abortion. Obstet Gynecol. 2014;123:676-692.

7. Winikoff B, Dzuba IG, Creinin MD, et al. Two distinct oral routes of misoprostol in mifepristone medical abortion: a randomized controlled trial. Obstet Gynecol. 2008;112:1303-1310.

8. Fiala C, Winikoff B, Helström L, et al. Acceptability of home-use of misoprostol in medical abortion. Contraception. 2004;70:387-392.

9. Kopp KH, Fiala C, Gemzell-Danielsson K. Assessment of significant factors affecting acceptability of home administration of misoprostol for medical abortion. Contraception. 2012;85:394-397.

10. National Abortion Federation. NAF Protocol for Mifepristone/Misoprostol in Early Abortion in the U.S. Washington, DC, 2010.

11. Jones $\mathrm{HE}, \mathrm{O}^{\prime}$ Connell WK, Norman WV, et al. First trimester medication abortion practice in the United States and Canada. PLoS One. 2017;12: e0186487.

12. U.S. Food and Drug Administration. Postmarket Drug Safety Information for Patients and Providers: Mifeprex (mifepristone) Information. Washington, DC, 2016.

13. Upadhyay UD, Johns NE, Combellick SL, et al. Comparison of outcomes before and after Ohio's law mandating use of the FDA-approved protocol for medication abortion: A retrospective cohort study. PLOS Med. 2016;13:e1002110.

14. Ohio Department of Health. Induced Abortions in Ohio: 2010. Columbus, $\mathrm{OH}, 2011$.

15. Ohio Department of Health. Induced Abortions in Ohio: 2011. Columbus, $\mathrm{OH}, 2012$.

16. Ohio Department of Health. Induced Abortions in Ohio: 2012. Columbus, $\mathrm{OH}, 2013$.

17. Ohio Department of Health. Induced Abortions in Ohio: 2013. Columbus, $\mathrm{OH}, 2014$.

18. Ohio Department of Health. Induced Abortions in Ohio: 2014. Columbus, $\mathrm{OH}, 2015$.

19. Sheldon WR, Winikoff B. Mifepristone label laws and trends in use: recent experiences in four U.S. states. Contraception. 2015;92: $182-185$.

20. Jones RK, Jerman J. Abortion incidence and service availability in the United States, 2011. Perspect Sex Reprod Health. 2014;46:3-14.

21. Jones RK, Jerman J. Abortion incidence and service availability in the United States, 2014. Perspect Sex Reprod Health. 2017;49:17-27.

22. Shochet T, Trussell J. Determinants of demand: method selection and provider preference among US women seeking abortion services. Contraception. 2008;77:397-404.

23. Ho PC. Women's perceptions on medical abortion. Contraception. 2006; 74:11-15.

24. Henshaw RC, Naji SA, Russell IT, et al. Comparison of medical abortion with surgical vacuum aspiration: women's preferences and acceptability of treatment. BMJ. 1993;307:714-717. 
25. Moreau C, Trussell J, Desfreres J, et al. Medical vs. surgical abortion: the importance of women's choice. Contraception. 2011;84:224-229.

26. Harvey SM, Beckman LJ, Satre SJ. Choice of and satisfaction with methods of medical and surgical abortion among U.S. clinic patients. Fam Plann Perspect. 2001;33:212-216.

27. Teal SB, Harken T, Sheeder J, et al. Efficacy, acceptability and safety of medication abortion in low-income, urban Latina women. Contraception. 2009;80:479-483.

28. Clark S, Ellertson C, Winikoff B. Is medical abortion acceptable to all American women: the impact of sociodemographic characteristics on the acceptability of mifepristone-misoprostol abortion. J Am Med Womens Assoc. 2000;55(3 Suppl):177-182.

29. Winikoff B, Ellertson C, Elul B, et al. Acceptability and feasibility of early pregnancy termination by mifepristone-misoprostol. Results of a large multicenter trial in the United States. Mifepristone Clinical Trials Group. Arch Fam Med. 1998;7:360-366.

30. Dehlendorf C, Rodriguez Ml, Levy K, et al. Disparities in family planning Am J Obstet Gynecol. 2010;202:214-220.

31. Jerman J, Jones RK, Onda T. Characteristics of U.S. Abortion Patients in 2014 and Changes Since 2008. New York: Guttmacher Institute, 2016.

32. Jatlaoui TC, Ewing A, Mandel MG, et al. Abortion surveillance-United States, 2013. MMWR Surveill Summ. 2016;65:1-44.

33. Finer LB, Zolna MR. Declines in unintended pregnancy in the United States, 2008-2011. N Engl J Med. 2016;374:843-852.

34. Kim TY, Dagher RK, Chen J. Racial/ethnic differences in unintended pregnancy: evidence from a national sample of U.S. women. Am J Prev Med. 2016:50:427-435.

35. Thorburn S, Bogart LM. Conspiracy beliefs about birth control: barriers to pregnancy prevention among African Americans of reproductive age. Health Educ Behav. 2005;32:474-487.

36. Harris LH, Wolfe T. Stratified reproduction, family planning care and the double edge of history. Curr Opin Obstet Gynecol. 2014;26 539-544.

37. Stern AM. Sterilized in the name of public health: race, immigration, and reproductive control in modern California. Am J Public Health. 2005;95: 1128-1138.
38. Schoen J. Choice \& Coercion: Birth Control, Sterilization, and Abortion in Public Health and Welfare. Chapel Hill, NC: University of North Carolina Press, 2005.

39. Roberts D. Killing the Black Body: Race, Reproduction, and the Meaning of Liberty. New York: Vintage, 2014.

40. University of Michigan Population Studies Center. Zip Code Characteristics: Mean and Median Household Income. Ann Arbor, MI, 2010.

41. Finer LB, Frohwirth LF, Dauphinee LA, et al. Timing of steps and reasons for delays in obtaining abortions in the United States. Contraception. 2006;74:334-344.

42. Guttmacher Institute. State Policies in Brief: An Overview of Abortion Laws. New York, NY, 2017.

43. Gerdts C, Fuentes L, Grossman D, et al. Impact of clinic closures on women obtaining abortion services after implementation of a restrictive law in Texas. Am J Public Health. 2016;106:857-864.

44. Reproductive Health Access Project. Mifepristone/misoprostol abortion protocol. New York, NY, 2016

Cite this article as: Upadhyay UD, Johns NE, Cartwright AF, Franklin TE (2018) Sociodemographic characteristics of women able to obtain medication abortion before and after Ohio's law requiring use of the Food and Drug Administration protocol, Health Equity 2:1, 122-130, DOI: 10.1089/heq.2018.0002.

\begin{aligned} \multicolumn{1}{c}{ Abbreviations Used } \\ $\mathrm{BMI}=$ body mass index \\ $\mathrm{FDA}=$ Food and Drug Administration \\ $\mathrm{GED}=$ general educational development \\ $\mathrm{ITOP}=$ Induced Termination of Pregnancy \\ $\mathrm{LMP}=$ last menstrual period \\ $\mathrm{N} . \mathrm{S} .=$ not statistically significant \end{aligned}

